\title{
ARTICLE \\ Gasdermin E-derived caspase-3 inhibitors effectively protect mice from acute hepatic failure
}

\author{
Wan-feng $\mathrm{Xu}^{1}{ }^{1}$, Quan Zhang ${ }^{1}$, Chu-jie Ding ${ }^{1}$, Hui-yong Sun ${ }^{1}$, Yuan $\mathrm{Che}^{1}$, Hai Huang ${ }^{1}$, Yun Wang ${ }^{1}$, Jia-wei Wu ${ }^{1}$, Hai-ping Hao ${ }^{1}$ and \\ Li-juan Cao ${ }^{1}$
}

Programmed cell death (PCD), including apoptosis, apoptotic necrosis, and pyroptosis, is involved in various organ dysfunction syndromes. Recent studies have revealed that a substrate of caspase-3, gasdermin E (GSDME), functions as an effector for pyroptosis; however, few inhibitors have been reported to prevent pyroptosis mediated by GSDME. Here, we developed a class of GSDME-derived inhibitors containing the core structure of DMPD or DMLD. Ac-DMPD-CMK and Ac-DMLD-CMK could directly bind to the catalytic domains of caspase-3 and specifically inhibit caspase-3 activity, exhibiting a lower IC ${ }_{50}$ than that of Z-DEVD-FMK. Functionally, Ac-DMPD/DMLD-CMK substantially inhibited both GSDME and PARP cleavage by caspase-3, preventing apoptotic and pyroptotic events in hepatocytes and macrophages. Furthermore, in a mouse model of bile duct ligation that mimics intrahepatic cholestasis-related acute hepatic failure, Ac-DMPD/DMLD-CMK significantly alleviated liver injury. Together, this study not only identified two specific inhibitors of caspase-3 for investigating PCD but also, more importantly, shed light on novel lead compounds for treating liver failure and organ dysfunctions caused by PCD.

Keywords: caspase-3 inhibitor; gasdermin E; apoptosis; pyroptosis; acute hepatic failure

Acta Pharmacologica Sinica (2021) 42:68-76; https://doi.org/10.1038/s41401-020-0434-2

\section{INTRODUCTION}

Multiple organ failure (MOF) is the most serious complication of trauma [1, 2], infection and other diseases with diverse etiologies $[3,4]$ and has complicated pathogenesis and high mortality. Acute hepatic failure refers to a group of hepatocyte dysfunctions characterized by massive loss of parenchymal cells, which is usually accompanied by severe synthetic and metabolic disorders, including jaundice, hepatic encephalopathy, and ascites [5-7]. Programmed cell death (PCD) plays a key role in mediating hepatocyte death. In addition to the well-known apoptosis, pyroptosis has emerged as a type of highly inflammatory cell death that is mainly responsible for immunocyte death and can also occur in hepatocytes [8]. Thus, targeting multiple forms of PCD might offer novel opportunities for therapeutic intervention in acute hepatic failure.

Pyroptosis is a new type of PCD initiated through pathogenassociated molecular patterns and mediated by inflammatory caspases, including caspase-1, -4, -5, and -11 [9-11]. Notably, pyroptosis is characterized by osmotic swelling, cell rupture, and release of inflammatory cytokines [12], while apoptosis is generally characterized by cell shrinkage, nuclear condensation, and exposure of phosphatidylserine, but membrane integrity is maintained in the early phase [13]. Gasdermin D (GSDMD), activated by inflammatory caspase- $1,-4$, and -11 , is a key executor of pyroptosis that targets the cell membrane and eventually leads to pore formation [9-11]. Interestingly, another protein of the gasdermin family, DFNA5/gasdermin E (GSDME), could be specifically cleaved by caspase- 3 at the $\mathrm{Asp}_{270}$ site, also generating an N-terminal fragment that targets the plasma membrane to induce pyroptosis $[14,15]$. Together, these studies suggest a link between apoptosis and pyroptosis, expanding the scope of pyroptosis from innate immunity to various types of tissue damage and chemotherapies under conditions of caspase-3 activation; thus, targeting pyroptosis may be a potential strategy for treating organ failure.

The GSDMD-derived inhibitor Ac-FLTD-CMK has been reported to suppress pyroptosis downstream of inflammasomes such as caspases-1, $-4,-5$, and -11 but not caspase-3 [16]. As a key executor of signal transduction, caspase- 3 plays crucial roles in cell death and tissue damage [17-19]. Several caspase-3 inhibitors have been developed and are effective in animal models of ischemic injury, burns, endotoxemia, and neonatal hypoxia [20, 21]. For example, the pancaspase inhibitor Z-VAD-FMK is potent but has poor specificity $[22,23]$. The peptide DEVD-based inhibitor AcDEVD-CHO is specific for caspase-3 but has poor cell activity and stability in vivo [24], and Z-DEVD-FMK appears to inhibit other caspases, such as caspase- $2,-7,-8$, and -10 [25]. In addition, the design of canonical caspase- 3 inhibitors is based on the cleavage site peptide of PARP, which is known to be involved in the execution of apoptosis. Whether such inhibitors show an inhibitory effect on pyroptosis remains unknown. For determination of the importance of caspase-3 activation in various forms of cell death, including apoptosis and pyroptosis, potent, selective, and multifunctional inhibitors are highly desirable.

${ }^{1}$ State Key Laboratory of Natural Medicines, Key Laboratory of Drug Metabolism, China Pharmaceutical University, Nanjing 210009, China

Correspondence: Hai-ping Hao (haipinghao@cpu.edu.cn) or Li-juan Cao (caolijuan0702@cpu.edu.cn)

The first two authors contributed equally: Wan-feng Xu, Quan Zhang

Received: 6 January 2020 Accepted: 9 May 2020

Published online: 26 May 2020 
In the present study, we demonstrated that the human/mouse GSDME cleavage site peptide DMPD/DMLD can directly bind to the catalytic domains of caspase-3. The GSDME-derived inhibitor Ac-DMPD/DMLD-CMK significantly suppressed the activation of caspase-3 as well as the downstream effectors PARP and GSDME, protecting cells from both apoptotic and pyroptotic cell death. Furthermore, in a mouse model of acute hepatic failure induced by bile duct ligation (BDL), Ac-DMPD/DMLD-CMK significantly reduced the serum lactate dehydrogenase (LDH) release and alanine aminotransferase/aspartate aminotransferase (ALT/AST) activities, alleviating hepatocyte injury. Moreover, GSDME derivatives showed a stronger direct inhibition of caspase-3 activity and cell death than PARP-derived Z-DEVD-FMK. Thus, this study reports two GSDME-derived inhibitors specific for caspase-3, which can be used for investigating caspase-3-mediated apoptosis and pyroptosis and are lead compounds for the treatment of liver failure and organ dysfunction induced by PCD.

\section{MATERIALS AND METHODS}

Reagents

DMPD/DMLD, DEVD, LEVD, IETD, and LEHD peptides were all synthesized by GenScript (Nanjing, China). Ac-DMPD/DMLD-CMK (982478/982479), biotin-C6-DMPD/DMLD-NH 2 (982482/982483), and Ac-DMPD/DMLD-AMC (982480/982481) were all synthesized by the Chinese Peptide Company (Hangzhou, China). LPS (L2630), deoxycholic acid (DCA, D2510), and cycloheximide (CHX, C7698) were purchased from Sigma-Aldrich (St Louis, MO, USA). Actinomycin D (ActD, HY17559), Z-DEVD-FMK (HY-12466), and Z-IETD-FMK (HY-101297) were obtained from MCE (Monmouth Junction, NJ, USA). Z-LEHD-FMK (S7313) was purchased from Selleck (Houston, TX, USA). Fugene/HD (E231A) was purchased from Promega (Madison, WI, USA), and recombinant human-TNFa (300-01 A) was purchased from Peprotech (Rocky Hill, CT, USA). Recombinant human active caspase-3-GFP was ordered from BioGot (Nanjing, China), and active caspase-4-GFP was ordered from DeTai Bio (Nanjing, China). Recombinant human active caspase-8 (ab198070) and active caspase-9 protein (ab198061) were purchased from Abcam (Cambridge, UK).

Animals and treatments

Wild-type male C57BL/6 J mice (Institute for Laboratory Animal Research: strain code: 219) were purchased from Beijing Vital River Laboratory Animal Technology Co., Ltd. (Beijing, China) at the age of 5 weeks $(20-22 \mathrm{~g})$. Animals were maintained at the Animal Facility according to protocols of the Review Committee of Animal Care and Use. All animal experiments were conducted with sexand age-matched mice and performed with approval from the China Pharmaceutical University Animal Care and Use Committee.

BDL model: Mice were anesthetized with chloral hydrate solution $(0.3 \mathrm{~g} / \mathrm{kg})$. A median abdominal incision was made to find the common bile duct. The duct was carefully ligated with 7-0 Prolene (Ethicon, Somerville, MA, USA) and dissected without ligation in the sham operation. The incision was wiped with an alcohol swab, and $0.5 \mathrm{~mL}$ of $0.9 \%$ saline was injected into the incision to improve recovery and survival. The mice were then used for experiments 5 days after surgery. Inhibitors $(5 \mathrm{mg} / \mathrm{kg}$ body weight) were intraperitoneally injected into the mice daily after surgery, and the same volume of sterile saline was injected into the sham and BDL model control groups. The serum ALT/AST levels were detected with ALT/AST Detection Kits (C009-2-1/ C0010-2-1, Nanjing Jiancheng Bioengineering Institute, Nanjing, China).

Cell culture and treatments

HepG2 (HB-8065, ATCC) and L-929 (CCL-1, ATCC) cells were grown in DMEM (Gibco, Thermo Fisher Scientific, Waltham, MA, USA) containing $10 \%$ fetal bovine serum (FBS), 2 mM L-glutamine, penicillin $(50 \mathrm{U} / \mathrm{mL})$ and streptomycin $(100 \mathrm{mg} / \mathrm{mL})$. Bone marrowderived macrophages (BMDMs) were isolated from C57BL/6 J mice as previously described $[26,27]$ and cultured in DMEM medium containing $10 \%$ FBS and $20 \%$ L-929 conditional medium. All cells were maintained in $5 \% \mathrm{CO}_{2}$ at $37^{\circ} \mathrm{C}$ and were excluded from endotoxin and mycoplasma by following the instructions of the Toxin Sensor $^{\mathrm{TM}}$ limuloid reagent detection kit (L00350, GenScript) and Mycoplasma Detection Kit (CA1080, Solarbio, Wuhan, China).

Distinct cell death models were established as previously described [14]. HepG2 cells were pretreated with $10 \mu \mathrm{g} / \mathrm{mL}$ CHX or $50 \mu \mathrm{g} / \mathrm{mL}$ ActD for $30 \mathrm{~min}$ and then synergistically challenged with $20 \mathrm{ng} / \mathrm{mL}$ TNFa for $16 \mathrm{~h}$ to induce apoptotic/pyroptotic cell death. HepG2 or BMDMs were challenged with $200 \mu M$ DCA for $4 \mathrm{~h}$ to mimic cholestasis-related acute hepatic injury. As a positive control of caspase-4/11-mediated pyroptosis, cells were primed with LPS $(1 \mu \mathrm{g} / \mathrm{mL})$ for $12 \mathrm{~h}$, and then, $5 \mu \mathrm{g} / \mathrm{mL}$ of LPS and $0.3 \%$ $(v / v)$ Fugene/HD were simultaneously suspended in $500 \mu \mathrm{L}$ of Opti-MEM medium for $30 \mathrm{~min}$. The suspensions were brought up to $3 \mathrm{~mL}$ with DMEM medium and incubated with cells in a 6-well plate (80\% confluence) for an additional $16 \mathrm{~h}$.

\section{Molecular docking}

The crystal structure of caspase-3 (PDB code: $2 \mathrm{~J} 30$ [28]) was used for molecular docking, where the position of the cocrystallized ligand (Ac-DEVD-CMK) was set as the binding site. Autodock 4.2 [29] with the Lamarckian genetic algorithm [30] was employed for molecular docking due to its good performance in reproducing the binding mode of the cocrystallized ligands to their targets [31-33]. Docking parameters: the RMSD of the reproduced cocrystallized ligand (gray sticks model) is $1.08 \AA$ compared with the crystallized ligand (green sticks model); a Gasteiger partial charge [34] was used for the electrostatic interaction calculation; and the docking space was set as $18.75 \AA \times 18.75 \AA \times 18.75 \AA$ (corresponding to $50 \times 50 \times 50$ grids, with each grid $0.375 \AA$ in length). All the ligands were docked 100 times for sufficient sampling, and the validation of docking structures is presented in Supplementary Fig. S1.

Microscale thermophoresis (MST) assay

Analyses of ligand binding were performed with an MST instrument (Nano Temper Technology, Munich, Germany). Inhibitors or peptides were used at 3.125, 6.25, 12.5, 25, 50, and $100 \mu \mathrm{M}$, and LPS was used at $0.06,0.12,0.24,0.48,0.96$, and $1.92 \mu \mathrm{M}$. GFP-tagged caspase-3/-4 or caspase-8/-9 (0.2 $\mu \mathrm{M})$ tagged with dye provided by the kit (MO-L004, Nano Temper Technology) was incubated with inhibitors or LPS for $30 \mathrm{~min}$ at room temperature. The mixture was inhaled with capillaries and placed on a specific measurement frame. The sample was detected under blue excitation mode with $40 \%$ LED power and $80 \%$ MST power by using NT Control software. Data were analyzed by using NT Analyses Version 1.5.41 software. Nonlinear curve-fitting of the binding was performed to calculate the dissociation constant $K_{\mathrm{d}}$.

\section{Streptavidin pulldown assay}

HepG2 cells were challenged as indicated and lysed with NP-40 lysis buffer containing $1 \times$ protease inhibitor cocktail (PIC, P8340, Sigma-Aldrich). One milliliter of lysis buffer containing $1 \mathrm{mg}$ of protein was incubated with $200 \mu \mathrm{M}$ biotin-C6-DMPD/DMLD or biotin-C6 overnight at $4{ }^{\circ} \mathrm{C}$ with constant rotation. Streptavidin magnetic beads (65001, Invitrogen, Carlsbad, CA, USA) were prepared in advance. In brief, $100 \mu \mathrm{L}$ of beads per group was washed three times with PBST before use and then added to a biotin-protein lysis buffer mixture to perform the standard pulldown assay for $6 \mathrm{~h}$ with constant rotation. The bead-biotinprotein mixture was washed three times with PBST using a magnetic separator, and the precipitates were then boiled in $2 \times$ SDS sample buffer followed by immunoblotting analysis. 
Caspase activity assay

For analysis of the potency of Ac-DMPD/DMLD-CMK against caspases, the following substrates were used: Z-VAD-AMC (I-1710.0005, BACHEM, Bubendorf, Switzerland) for caspase-3, ACLEVDAMC (ALX-260-083-M005, Enzo, Farmingdale, NY, USA) for caspase-4, AC-IETD-AMC (556552, BD Pharmingen, San Diego, CA, USA) for caspase-8 and Ac-LEHD-AMC (AAT-13426, AAT Bioquest, Inc., Sunnyvale, CA, USA) for caspase-9. The inhibitor was preincubated with $50 \mathrm{nM}$ active caspase for $2 \mathrm{~h}$ at $25^{\circ} \mathrm{C}$ in $100 \mu \mathrm{L}$ of reaction buffer (50 mM HEPES (pH 7.5), 1 mM EDTA, 10 mM DT, $0.1 \%$ CHAPS, $100 \mathrm{mM} \mathrm{NaCl}$, and $10 \%$ glycerol), and then, $200 \mu \mathrm{M}$ substrates were added. Caspase activities were monitored at $37^{\circ} \mathrm{C}$ with Ex $365 \mathrm{~nm}$ and Em $450 \mathrm{~nm}$ for AMC hydrolysis detection. A dose-response curve was obtained by testing inhibitors in a series of concentrations as indicated in the figure legends. $I C_{50}$ values were calculated by using GraphPad Prism 6.0.

For determination of the activities of caspase- 3 substrates designed in this paper, 6.25-200 $\mu \mathrm{M}$ Ac-DMPD/DMLD-AMC was incubated with $50 \mathrm{nM}$ recombinant human active caspase- 3 at $37^{\circ} \mathrm{C}$ for $30 \mathrm{~min}$, and the fluorescence intensity was monitored as above. Caspase- 3 activities are shown as relative values to that of the vehicle.

For determination of caspase-3 activity in the livers of mice, $10 \mathrm{mg}$ tissue was homogenized with lysis buffer containing DTT and centrifuged at $13,000 \times g$ for $10 \mathrm{~min}$, and the supernatant was collected to determine the protein concentrations. Homogenate containing $150 \mu \mathrm{g}$ of protein was used for caspase-3 activity assay, by incubating with $200 \mu \mathrm{M}$ of the substrate Z-VAD-AMC at $37^{\circ} \mathrm{C}$ for $30 \mathrm{~min}$ in a $100 \mu \mathrm{L}$ reaction system. The fluorescence intensity was detected as above, and normalized caspase- 3 activity is shown as the relative value to that of the sham group.

\section{Microscopy}

For analysis of the morphology of apoptotic and pyroptotic cells, cells were seeded in cover-glass bottom dishes at $40 \%$ confluency and treated as indicated in the figure legends. Then, cell images were captured using an LSM700 confocal microscope in DIC mode (Zeiss, Oberkochen, Germany).

\section{Flow cytometry}

For flow cytometry analysis, cells were treated as in the cytotoxicity assays. In brief, approximately $1 \times 10^{6}$ cells per group were collected and centrifuged at $1500 \mathrm{rpm}$ for $5 \mathrm{~min}$. Each group was stained with $200 \mu \mathrm{L}$ of $1 \times$ binding buffer containing $2.5 \mu \mathrm{L}$ of Annexin V-FITC and $2.5 \mu \mathrm{L}$ of propidium iodide (PI, 556547, BD Pharmingen). The cells were resuspended and analyzed by a BD Accuri C6 plus flow cytometer (BD Biosciences, San Jose, CA, USA).

\section{SiRNA transfection}

HepG2 cells were transfected with CASPASE-3 siRNA (sc-29237) or negative control siRNA (sc-44230) from Santa Cruz Biotechnology (Dallas, TX, USA) by using a reverse transfection method according to the instructions. In detail, $500 \mu \mathrm{L}$ of Opti-MEM medium and $5 \mu \mathrm{L}$ of Lipofectamine ${ }^{\mathrm{TM}}$ RNAiMAX Transfection Reagent (13778150, Invitrogen) containing $20 \mathrm{nM}$ siRNA or $20 \mathrm{nM}$ negative control siRNA were premixed in a six-well plate. HepG2 cells were plated at a density of $5 \times 10^{5}$ per well, and the optimal transfection time was $48 \mathrm{~h}$, as the maximal silencing efficiency was shown by Western blot analysis.

\section{Western blotting}

Collected cells or homogenized tissue were lysed in RIPA solution containing PIC (P8340, Sigma-Aldrich). Protein contents in lysates were quantitated by the BCA assay kit (Beyotime Biotechnology, Shanghai, China), determined before the addition of sample buffer (Bio-Rad, Hercules, CA, USA) and boiled. A total of $60 \mu \mathrm{g}$ of protein was subjected to SDS-PAGE electrophoresis (Bio-Rad), transferred to $2.2 \mu \mathrm{m}$ polyvinylidene fluoride membranes (Bio-Rad) and probed with primary antibodies followed by HRP-conjugated secondary antibodies. Antibodies including GSDME (ab215191) and GAPDH (ab8245) were purchased from Abcam, caspase-3 (9662), and PARP (9532) antibodies were purchased from Cell Signaling Technology (Boston, MA, USA), and caspase-4 (sc-56056) antibody was purchased from Santa Cruz Biotechnology. HRPconjugated antirabbit lgG $(\mathrm{H}+\mathrm{L})$ antibody (ab6721) and HRPconjugated antimouse $\lg (\mathrm{H}+\mathrm{L})$ antibody (ab6789) were purchased from Abcam. The immunoreactive bands were visualized with HRP substrate (170-5061, Bio-Rad) by using an iBright CL1000 System (Invitrogen). GAPDH was immunoblotted as an internal control. For Western blot analysis of tissue samples, tissue homogenates from six mice were merged by three-in-one into two merged samples.

\section{Statistical analysis}

Data are presented as the mean \pm SEM and representative of at least two independent experiments; the number in each group $(n)$ is stated in the figure legends. Image data of the in vitro experiments are representative of triplicate samples per group, and image data of the in vivo experiments are representative of six mice per group. Statistical significance was determined by oneway ANOVA. $P<0.05$ was considered significant. All statistical analyses were performed with GraphPad Prism 6.0. For animal studies, the sample sizes were based on the empirically assessed variability of the model systems or assays used, and groups contained no less than six mice to minimize type I/II errors.

\section{RESULTS}

GSDME-derived inhibitors specifically target caspase-3

Previous studies identified two caspase-3-cleavable sites, ${ }_{267} \mathrm{DMPD}_{270}$ in human GSDME and ${ }_{267} \mathrm{DMLD}_{270}$ in mouse GSDME $[14,35]$. We designed two GSDME derivatives by linking the DMPD and DMLD peptides to the chloro-methyl ketone (-CMK) group to produce competitive inhibitors targeting caspase-3, named AcDMPD/DMLD-CMK (Fig. 1a, b). For elucidation of the engagement of the inhibitors in caspase-3, a crystal structure of the catalytic domain of human caspase-3 (PDB ID code: 2 J30) was docked with inhibitors. Z-DEVD-FMK was included as a positive control that was previously derived from the restriction site of PARP (Fig. 1c-e). Extensive hydrophilic and hydrophobic interactions were detected between the inhibitors and caspase-3. As previously described [36], Z-DEVD-FMK formed hydrogen bonds with R64 and R207 from caspase-3 (Fig. 1c). Interestingly, Ac-DMLD-CMK also formed hydrogen bonds with R207 and F250 from caspase-3 (Fig. 1d), while Ac-DMPD-CMK formed contacts with R207, S205, and S209 (Fig. 1e). Caspase-3 has four active site loops, the L1-L4 loops [28], and the analyses of binding residues indicated that all three inhibitors occupied the L3 (residues 198-213) and L4 (residues 247-263) loops, comprising the sides of the active sites in caspase3 . These results indicated that these inhibitors may share the same catalytic groove.

The direct binding of the inhibitors with the human active caspase- $3,-4,-8$, and -9 proteins was further confirmed by MST analysis; LPS for caspase-4 [10] and commercial inhibitors for caspase-3, -8 , and -9 were included as positive control molecules. As shown in Fig. 1f, compared with Z-DEVD-FMK, which showed a $K_{d}$ value of $2.6 \mu \mathrm{M}$ in binding with caspase-3, both Ac-DMPD-CMK and Ac-DMLD-CMK could bind with caspase- 3 with lower $K_{d}$ values of $1.62 \mu \mathrm{M}$ and $1.48 \mu \mathrm{M}$, respectively (Fig. $1 \mathrm{~g}$ ), and no significant binding was observed with human caspase-4, -8 , and -9 . To exclude the effects of CMK or FMK modifications, we incubated the DMLD/ DMPD/DEVD peptides with recombinant human caspases, and the $K_{\mathrm{d}}$ value was measured by MST assays. Consistently, DMPD/DMLD showed a lower $K_{d}$ in binding with caspase-3 than DEVD (Supplementary Fig. S2). Moreover, peptides without modification showed a higher $K_{d}$ value in binding with caspase-3 than CMK 
a<smiles>CSCC[C@H](NC(=O)[C@H](CC(=O)O)NC(C)=O)C(=O)N1CCC[C@H]1C(=O)N[C@H](CC(=O)O)C(=O)CCl</smiles>

b

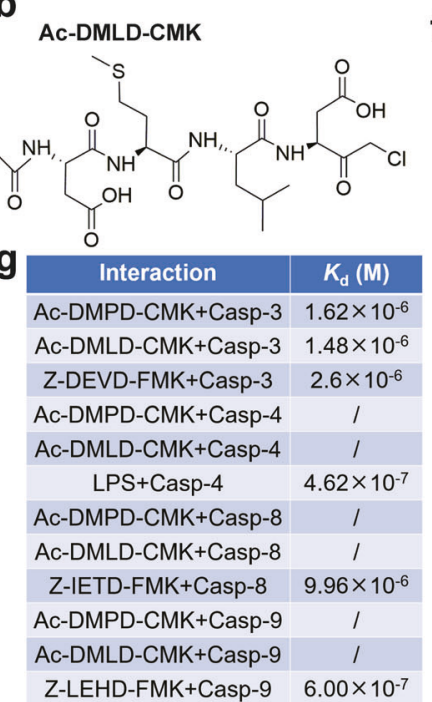

d

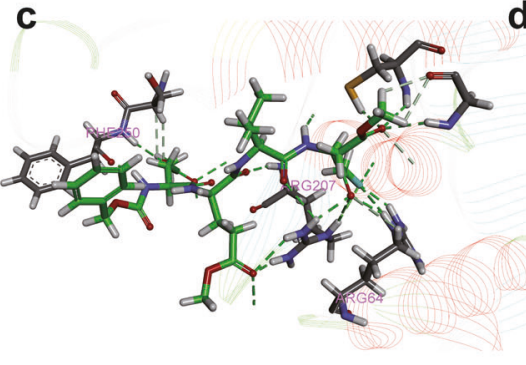

f

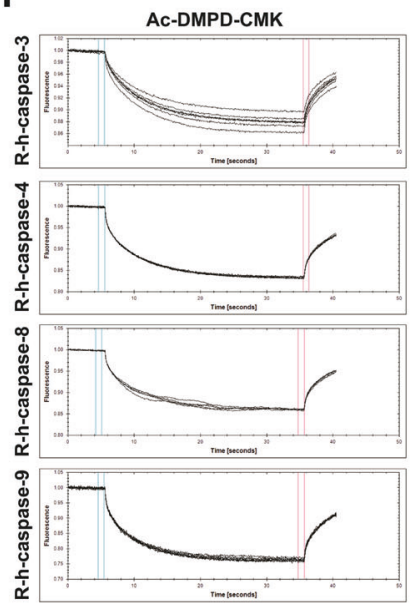

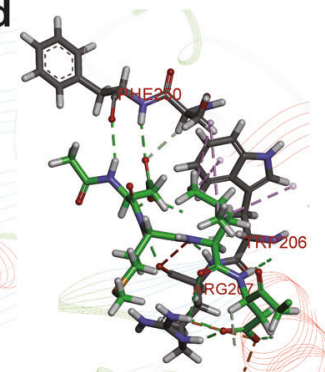

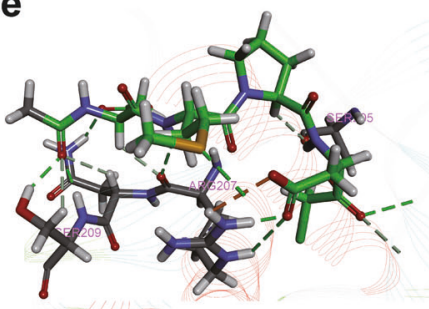

a !

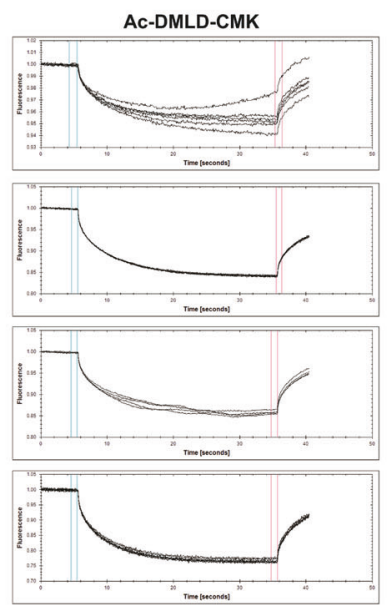

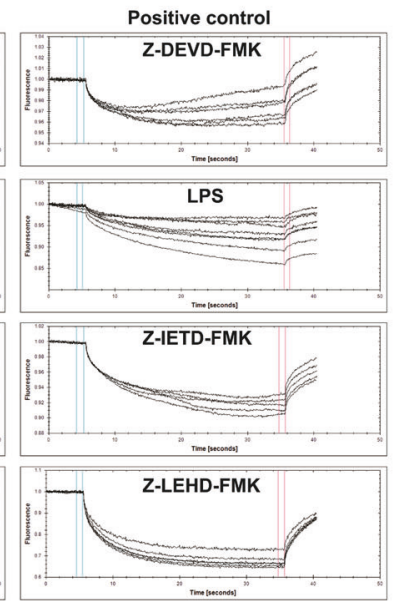

Positive control

Fig. 1 Ac-DMPD/DMLD-CMK specifically interacts with caspase-3. $\mathbf{a}$ and $\mathbf{b}$ Chemical structures of the human and mouse GSDME-derived inhibitors Ac-DMPD-CMK and Ac-DMLD-CMK. c-e Molecular docking between inhibitors as indicated and mature caspase-3 fragments; hydrogen bonds are indicated as green dotted lines. The inhibitors are shown in green sticks, and the caspase- 3 structure is shown in gray (PDB ID code: 2 J30). f MST analysis between inhibitors and human caspase- $3,-4,-8$, and -9 catalytic domains. $g$ Values of $K_{d}$ calculated from (f) are listed in the table.

conjugates. These data are consistent with a previous study showing that CMK or FMK had a covalent binding effect in addition to its stabilization effect [37]. Collectively, these data suggest direct and specific binding between Ac-DMPD/DMLD-CMK and caspase-3, consistent with previous findings that GSDME is only cleaved by caspase-3, rather than other caspases [14].

Ac-DMPD/DMLD-CMK specifically inhibits caspase-3

Next, to investigate the effects of Ac-DMPD/DMLD-CMK on caspase activities, we performed in vitro caspase activity assays by using synthetic caspase substrates. We observed that AcDMPD-CMK and Ac-DMLD-CMK potently inhibited the enzymatic activities of recombinant human active caspase-3 protein with $\mathrm{IC}_{50}$ values of $0.5456 \mu \mathrm{M}$ and $0.7455 \mu \mathrm{M}$, respectively. Ac-DMPD/DMLDCMK exhibited stronger efficacies than the commercial inhibitor ZDEVD-FMK, with an $\mathrm{IC}_{50}$ of $1.326 \mu \mathrm{M}$ (Fig. 2a-C), and nonspecific interactions with caspase-4, 8, and 9 were excluded (Fig. 2d-i). Furthermore, HepG2 cell lysates were incubated with active recombinant human caspase-3 protein, and cleavage of native GSDME and PARP was markedly inhibited by $20 \mu \mathrm{M}$ Ac-DMPDCMK, Ac-DMLD-CMK and the positive control Z-DEVD-FMK (Fig. 2j). These observations indicate that Ac-DMPD/DMLD-CMK are specific for caspase-3, which competitively inhibits the cleavage of both the apoptotic effector PARP and the pyroptotic effector GSDME. The PARP-derived inhibitor Z-DEVD-FMK acts much like GSDME-derived inhibitors, suggesting that they may occupy the same catalytic sites, further confirming the results of molecular docking.
Furthermore, biotinylated DMPD and DMLD (Fig. 3a) were designed and applied to investigate the binding between DMPD/ DMLD sites and native caspase-3 in cells. HepG2 cells were treated with TNFa/CHX and TNFa/ActD to induce caspase-3 activation, while LPS transfection was used to induce caspase-4 activation. Streptavidin pulldown assays demonstrated that the catalytic fragment of caspase-3 (p15) was pulled down by biotin-DMPD/ DMLD, while no obvious interaction of caspase-4 (p20) with biotinDMPD/DMLD was detected (Fig. 3b). To further identify the affinity, we designed the novel caspase-3 substrates Ac-DMPD/ DMLD-AMC (Fig. 3c) in this paper. With the increase in substrates incubated with recombinant human caspase-3, caspase-3 activities showed a linear correlation, which matched the first-order kinetic equation of enzymatic reactions (Fig. 3d, e). Together, these findings suggest that DMPD/DMLD peptides can bind with the caspase-3 catalytic domain.

Ac-DMPD/DMLD-CMK inhibit both pyroptosis and apoptosis Previous studies have indicated that some peptide inhibitors, such as Bz-DAVD-phenylpropyl ketone, had poor cell activity due to limited cell permeability [38]. To probe the inhibition of Ac-DMPD/ DMLD-CMK in cells, we studied the effects of these inhibitors on cell death induced by chemotherapy drugs. CHX or ActD synergistically treated with TNFa is a classical apoptosis inducer; however, recent studies have shown that cells highly expressing GSDME undergo pyroptosis upon "apoptotic stimulation," including chemotherapy drugs; [14] thus, these two chemotherapy drugs can simultaneously induce apoptotic and pyroptotic cell 

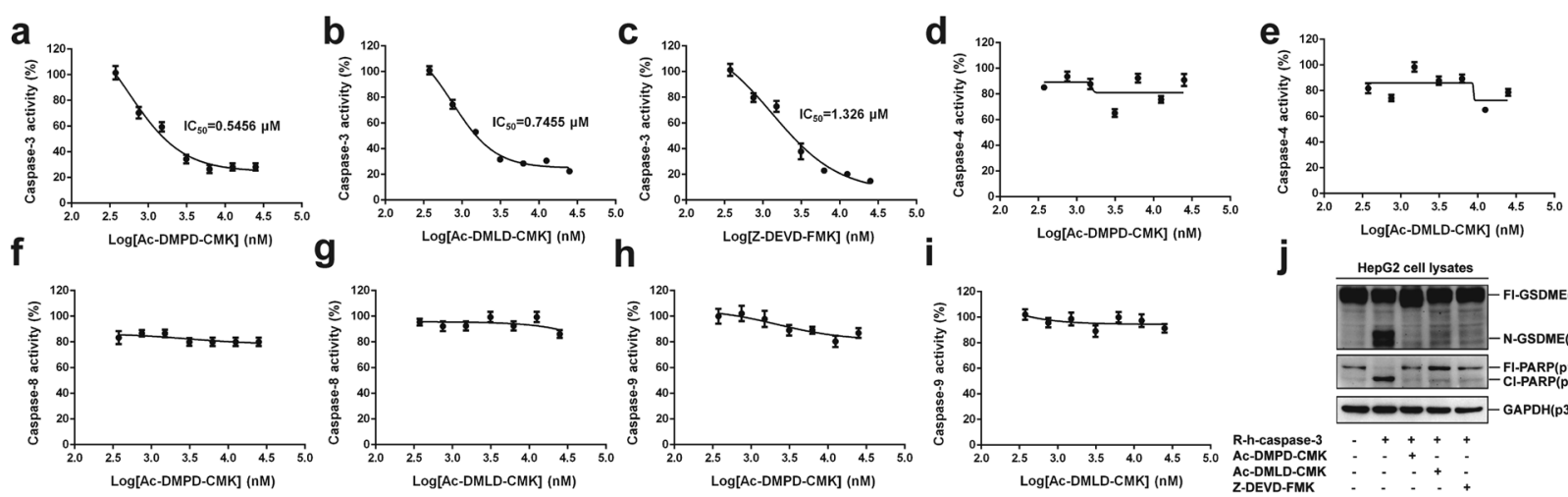

j

Log[AC-DMLD-CMK] (nM)

$$
\text { Log[AC-DMPD-CMK] (nM) }
$$

$$
\text { Log[Ac-DMLD-CMK] (nM) }
$$

Log[AC-DMPD-CMK] (nM)

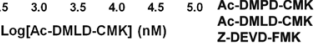

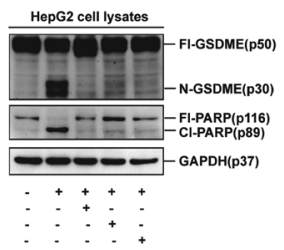

Fig. 2 Ac-DMPD/DMLD-CMK specifically inhibited caspase-3. a-i Relative enzymatic activities of human caspase-3, caspase-4, caspase-8, and caspase-9 in the presence of $0.375-25 \mu \mathrm{M}$ inhibitors as indicated. $\mathbf{j}$ Representative immunoblots of GSDME and PARP in HepG2 cells incubated with inhibitors as indicated and recombinant active caspase-3. Data are expressed as the mean $\pm \operatorname{SEM}(n=3)$.

a<smiles>CSCC[C@H](NC(=O)C(CC(=O)O)NC(=O)CCCCCNC(=O)CCCC[C@@H]1SC[C@@H]2NC(=O)N[C@H]12)C(=O)N1CCC[C@H]1NC(CC(=O)O)C(N)=O</smiles>

Biotin-C6-DMLD-NH2<smiles>CSCCC(NC(=O)CCC(=O)NC(=O)CCCCCNC(=O)CCCCC1SCCC1NC(=O)O)C(=O)N[C@@H](CC(=O)O)C(N)=O</smiles>

C Ac-DMPD-AMC<smiles>CSCC[C@H](NC(=O)C(CC(=O)O)NC(=O)[C@H](CC(=O)Nc1ccc2c(C)cc(=O)oc2c1)NC(C)=O)C(=O)N1CCCC1</smiles>

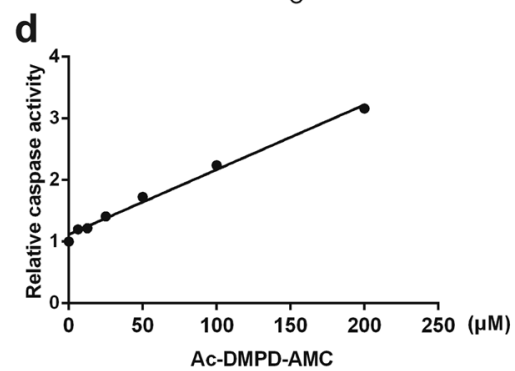

b

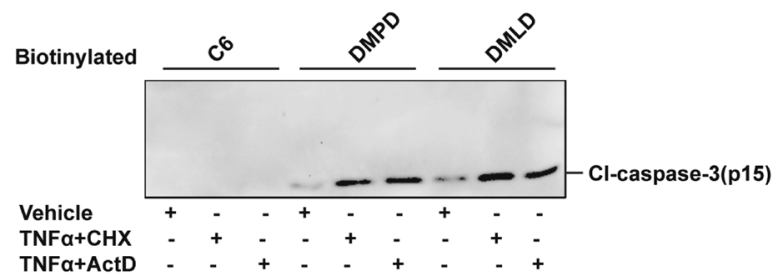

Input
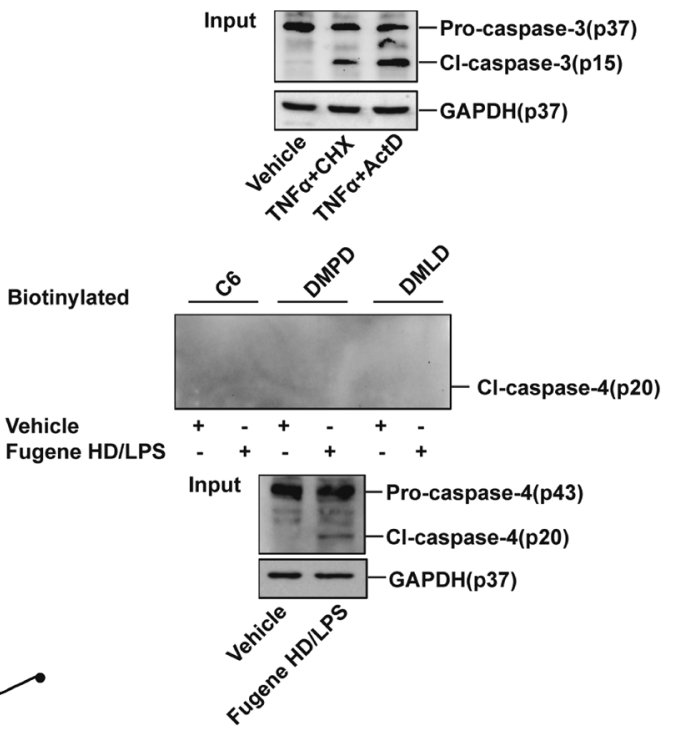

Fig. 3 The GSDME-derived peptides DMPD and DMLD directly bind to the caspase-3 catalytic domain. a Chemical structures of the biotinylated DMPD and DMLD. b Streptavidin pulldown assays of biotinylated DMPD/DMLD binding to native caspase-3 or -4 in HepG2 cells. c Chemical structures of the GSDME-derived fluorogenic substrates Ac-DMPD-AMC and Ac-DMLD-AMC. $\mathbf{d}$ and e Relative enzymatic activities of human active caspase-3 protein with Ac-DMPD-AMC (d) and Ac-DMLD-AMC (e). Data are expressed as the mean \pm SEM $(n=3)$.

death in HepG2 cells with high expression of GSDME [15]. The potency of the inhibitors against cytotoxicity was measured by $\mathrm{LDH}$ release assays. The results showed that both Ac-DMPD/ DMLD-CMK inhibited HepG2 cell death (Fig. 4a-f) more potently
$\left(I C_{50}\right)$ than the commercial inhibitor Z-DEVD-FMK. As predicted, cleavage of PARP or GSDME induced by chemotherapy drugs was concurrently blocked by Ac-DMPD/DMLD-CMK (Fig. 4g, h). To probe the specific effects of the two inhibitors on cell apoptosis 

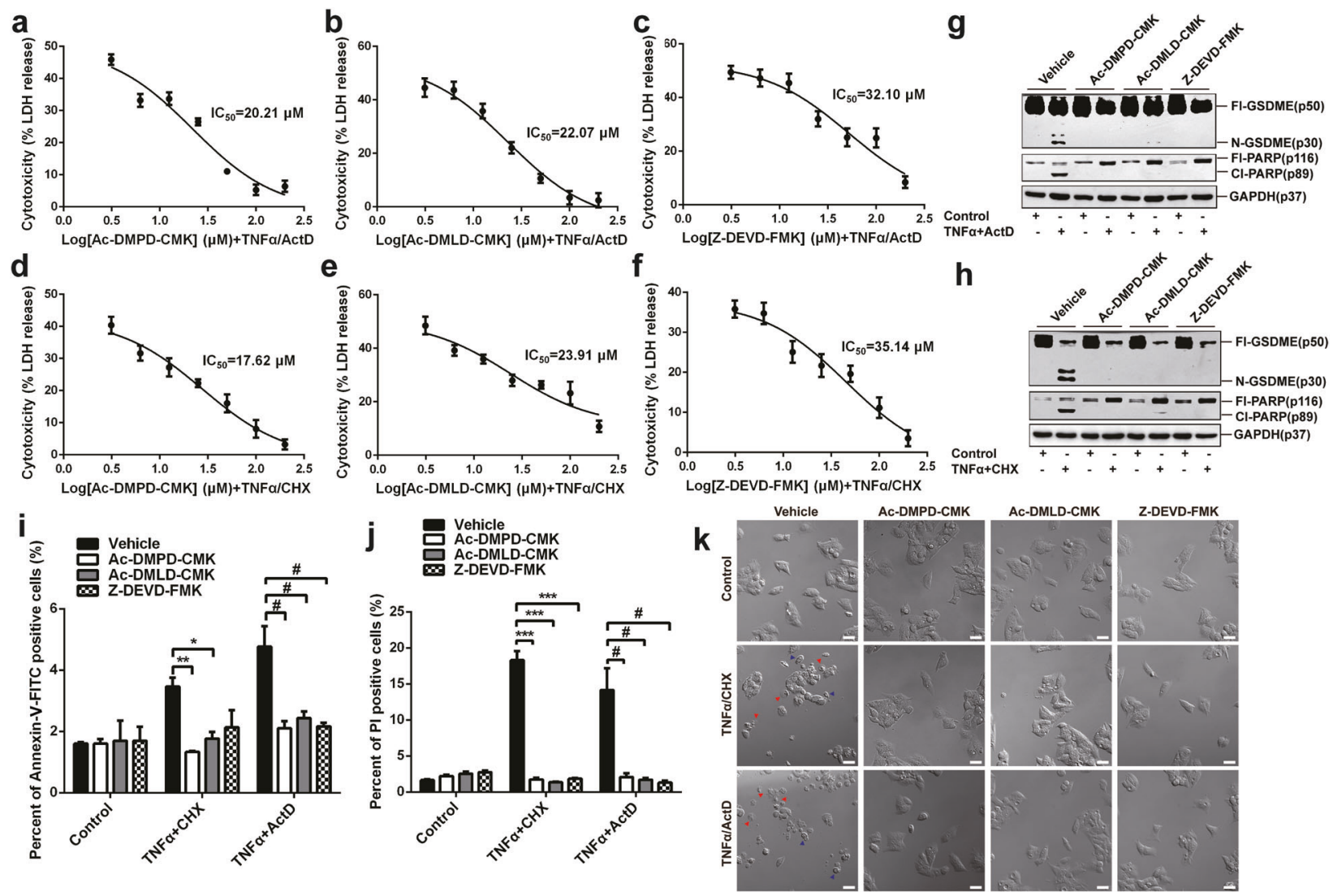

Fig. 4 Ac-DMPD/DMLD-CMK inhibit both pyroptosis and apoptosis. a-f LDH release assays for the cytotoxicity of HepG2 cells. Cells were treated with inhibitors at 3.125-200 $\mu \mathrm{M}$ for $2 \mathrm{~h}$, followed by TNF $\alpha / C H X$ or TNF $\alpha / A c t D$ for $16 \mathbf{h}$. $\mathbf{g}$ and $\mathbf{h}$ Representative immunoblots of GSDME and PARP in HepG2 cells. $\mathbf{i}$ and $\mathbf{j}$ Flow cytometry analysis of Annexin V-FITC- or PI-positive HepG2 cells. k Changes in HepG2 cell morphology were observed with a microscope (scale bar $=20 \mu \mathrm{m}$, magnification of the microphotograph, $\times 200$ ). Data are expressed as the mean \pm SEM $(n=3) .{ }^{*} P<0.05,{ }^{* *} P<0.01,{ }^{* *} P<0.001$ vs. the vehicle group of $\mathrm{TNF} \alpha / \mathrm{CHX} ;{ }^{*} P<0.05$ vs. the vehicle group of TNF $\alpha / A c t D$.

and pyroptosis, we quantified the Annexin V-FITC- and PI-positive cells with flow cytometry analysis (Supplementary Fig. S3). The statistical proportions of HepG2 cell death showed that both the apoptotic and pyroptotic rates were significantly decreased by AcDMPD/DMLD-CMK and Z-DEVD-FMK $(P<0.05, P<0.01, P<0.001$, respectively; Fig. $4 \mathrm{i}$, j). To further intuitively confirm that the two types of PCD were both blocked by inhibitors, we investigated the changes in HepG2 cell morphology. The results showed that cell shrinkage (apoptosis, blue arrows) and cell rupture (pyroptosis, red arrows) were both reversed (Fig. 4k). Together, our data suggest that Ac-DMPD/DMLD-CMK potently inhibits both apoptosis and pyroptosis at the cellular level.

\section{Ac-DMPD/DMLD-CMK protect against hepatic injury in mice with} BDL

Since the 1980s, with the development of medication therapy, mechanical assistance, and organ transplantation, treatments for single organ failure have substantially improved. However, many patients eventually die from MOF-like symptoms [3, 4, 39]. One of the most pressing issues of MOF is hepatic injury because of its importance in metabolism and detoxification. Acute hepatic failure is often accompanied by the loss of a large number of parenchymal cells and lacks effective clinical therapies [40, 41]. To further explore the in vivo activity of Ac-DMPD/DMLD-CMK, we next investigated the relevance of our in vitro findings in a mouse model of BDL, which could induce cholestasis and hepatocyte death and eventually mimic acute hepatic failure. Ac-DMPD/ DMLD-CMK or Z-DEVD-FMK $(5 \mathrm{mg} / \mathrm{kg})$ was intraperitoneally injected into the mice daily after BDL surgery for 5 days, and the serum levels of ALT/AST and LDH were all significantly reduced in the mice with $\mathrm{BDL}$ after treatment with Ac-DMPD/ DMLD-CMK, which was better than Z-DEVD-FMK as predicted $(P<$ $0.05, P<0.01, P<0.001$; Fig. 5a-c). We next explored the effects of the inhibitors on caspase- 3 activity in vivo. Caspase- 3 activity in liver tissue was entirely blocked by Ac-DMPD/DMLD-CMK, even decreasing to levels comparable to those in the sham group $(P<$ $0.05, P<0.01, P<0.001$; Fig. $5 \mathrm{~d}$ ). Moreover, representative H\&Estained sections of the liver showed that mice were effectively protected by Ac-DMPD/DMLD-CMK from hepatic injuries, as shown by alleviated parenchymal cell death and fibrosis (Fig. 5e). As predicted, immunoblot analysis showed that Ac-DMPD/DMLDCMK significantly inhibited the cleavage of GSDME and PARP in the livers of the mice with BDL (Fig. 5f). Collectively, these findings suggest that Ac-DMPD/DMLD-CMK act as promising inhibitors of caspase-3 activation in vivo.

\section{Ac-DMPD/DMLD-CMK inhibit cell death in hepatocytes and} macrophages induced by bile acid

To further validate the mechanism of Ac-DMPD/DMLD-CMK, we used DCA as a cytotoxicity inducer to mimic cholestatic liver injury, as the accumulation of DCA is a risk factor for hepatocyte damage and inflammation in cholestasis [42-44]. BMDMs were also included as model primary macrophages. All inhibitors counteracted the DCA-induced cell death in both HepG2 (Fig. 6a-c) and BMDMs (Fig. 6e-g) and concurrently inhibited GSDME and PARP cleavage (Fig. 6d) in HepG2 cells. Flow 

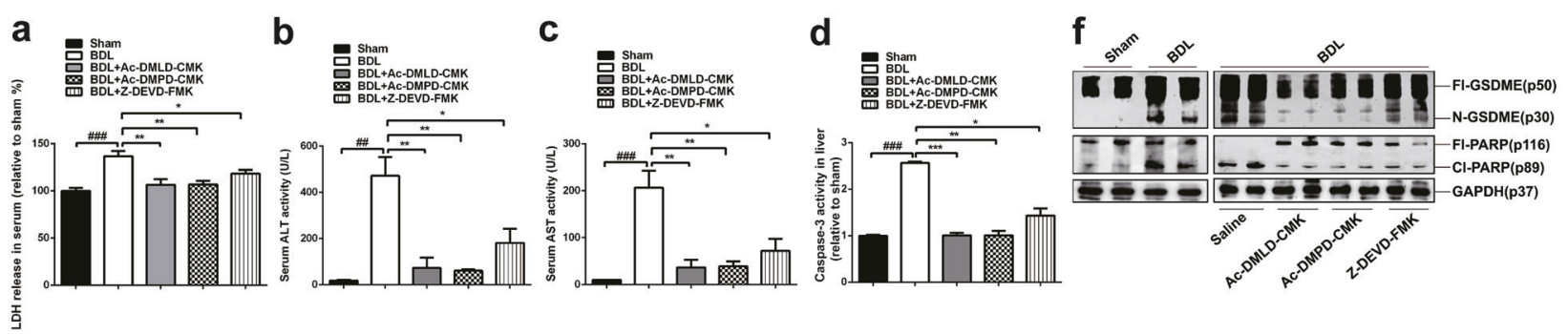

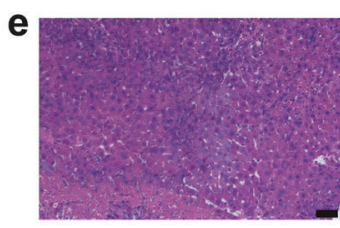

Sham

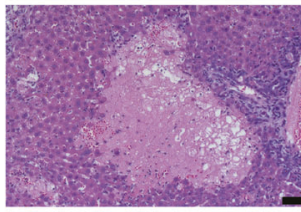

BDL

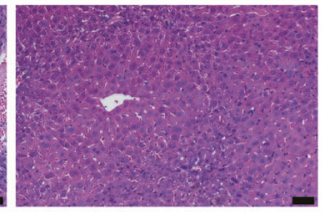

BDL+Ac-DMPD-CMK

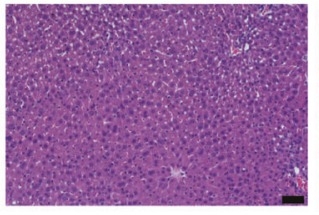

BDL+AC-DMLD-CMK

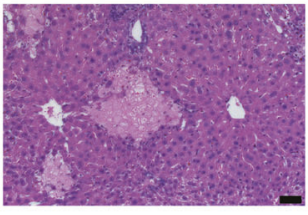

BDL+Z-DEVD-FMK

Fig. 5 Ac-DMPD/DMLD-CMK protects against hepatic injury in mice with bile duct ligation. a-c Serum LDH, ALT, and AST levels in the mice, $n=6$. d Caspase-3 activity in the liver tissue of the mice, $n=6$. e Representative H\&E sections of the liver (scale bar $=50 \mu \mathrm{m}, \mathrm{magnification}$ of the microphotograph, $\times 200)$. $f$ Representative immunoblots of GSDME and PARP in the livers of the mice. Data are expressed as the mean \pm SEM. ${ }^{*} P<0.05,{ }^{* *} P<0.01,{ }^{* * *} P<0.001$ vs. the BDL group; ${ }^{\# \#} P<0.01,{ }^{\# \# \#} P<0.001$ vs. the sham group.

cytometric analysis of Annexin V-FITC/PI staining and morphological changes of BMDMs indicated that both apoptosis and pyroptosis were completely blocked by these inhibitors $(P<0.01$, $P<0.001$, respectively; Supplementary Fig. S4 and Fig. $6 \mathrm{~h}-\mathrm{j})$. To further identify the effects of the inhibitors on caspase- 3 activity, we performed CASPASE-3 knockdown in HepG2 cells with siRNA (Fig. 6k). Cytotoxicity $(P<0.05, P<0.01$; Fig. $6 \mathrm{l})$, cell death $(P<0.05$, $P<0.01$; Supplementary Fig. S5 and Fig. $6 \mathrm{~m}, \mathrm{n})$ and morphological changes (Fig. 6o) were all blocked by the inhibitors, and similar results were observed in the CASPASE-3-silenced HepG2 cells. It appears that inhibition of caspase-3 by Ac-DMPD/DMLD-CMK can restrain DCA-induced apoptosis and pyroptosis in both hepatocytes and macrophages. Collectively, our study proposed a novel hepatoprotective strategy using GSDME-derived peptides directed against caspase-3-induced cell death in vitro and in vivo.

\section{DISCUSSION}

In recent years, an increasing number of patients has shown unexplained liver failure; $[45,46]$ this condition is difficult to predict and cure due to the rapid deterioration of liver function. Patients with different etiologies need specific therapeutic strategies, which increases the level of difficulty in treating liver failure $[47,48]$. However, a common pathological mechanism in hepatic failure is massive cell death, and excessive hepatocyte death amplifies inflammatory damage and promotes the development of fibrosis and ultimately liver failure [49, 50]. Thus, inhibiting the cell death of hepatocytes and immunocytes has been suggested to be a plausible therapeutic strategy for hepatic injury, especially acute hepatic failure. A number of studies are underway to develop inhibitory agents to target cell death signaling molecules, such as the mixed lineage kinase inhibitor CEP-1347 and poly (ADP-ribose) polymerase inhibitor for nigral dopaminergic neurons [51]. Caspase-3 plays a key role in cell death by activating the downstream effectors PARP and GSDME [14], and inhibition of caspase-3 can potently protect mice from ischemic injury, burns and endotoxemia $[17,21]$. Since GSDME has been shown to control cell fate in conditions of caspase-3 activation, the GSDME cleavage site may be a potent hepatoprotective therapeutic target.

Although GSDME is known as an effector of pyroptosis acting downstream of caspase-3, the development of specific and potent dual peptide inhibitors for caspase-3-induced apoptotic and pyroptotic cell death remains elusive in this field. The present study demonstrated interactions between caspase-3 and the GSDME-derived inhibitors Ac-DMPD/DMLD-CMK as well as peptides containing the cleavage site residues DMPD/DMLD. Our data suggest that the cell-permeable inhibitors Ac-DMPD/DMLD-CMK, directed against the caspase-3 cleavage site, effectively blocked caspase-3-dependent PARP and GSDME activation, dually protecting hepatocytes and macrophages from apoptotic and pyroptotic cell death. Moreover, Ac-DMPD/DMLD-CMK are more potent than the widely used caspase-3 inhibitor Z-DEVD-FMK. Notably, the DMPD/DMLD-derived inhibitors significantly alleviated extensive hepatic injury and reduced the serum ALT/AST and LDH levels in mice undergoing cholestasis. Collectively, our study proposed a novel hepatoprotective strategy using GSDME-derived peptides directed against caspase-3-induced cell death in vitro and in vivo.

In conclusion, we demonstrated that two novel cell-permeable inhibitors, Ac-DMPD-CMK and Ac-DMLD-CMK, can exert excellent protective effects against apoptotic and pyroptotic cell death in vitro and in vivo. However, further improvement is required because these inhibitors could potentially target other caspases at high concentrations, and more work is needed in the field of drug discovery against organ failure. Nonetheless, Ac-DMPD/DMLDCMK can be useful tools for studying the importance of caspase-3 activation in experimental systems and, more importantly, contribute to lead compound discovery for the treatment of organ failure.

\section{ACKNOWLEDGEMENTS}

This work was financially supported by the National Natural Science Foundation of China (HPH is supported by 81720108032,81930109 , and 81421005; and LC is supported by 81973559), the Project for Major New Drug Innovation and Development (grants 2018ZX09711001-002-003 to HPH; 2018ZX09711002-001-004 to LUC), the Overseas Expertise Introduction Project for Discipline Innovation (G20582017001) to HPH, the "Double-First Class" initiative project (CPU2018GF09) and the Sanming Project of Medicine in Shenzhen (grants SZSM201801060 to HPH). We thank the Chinese Peptide Company for designing synthetic routes and performing compound synthesis in this study.

\section{AUTHOR CONTRIBUTIONS}

LC and HPH designed the study; WFX, QZ, CJD, HYS, YC, HH, YW, and JWW performed the experiments and collected and analyzed the data; LC, HPH, and WFX wrote and revised the manuscript. WFX and QZ contributed equally to this work. 

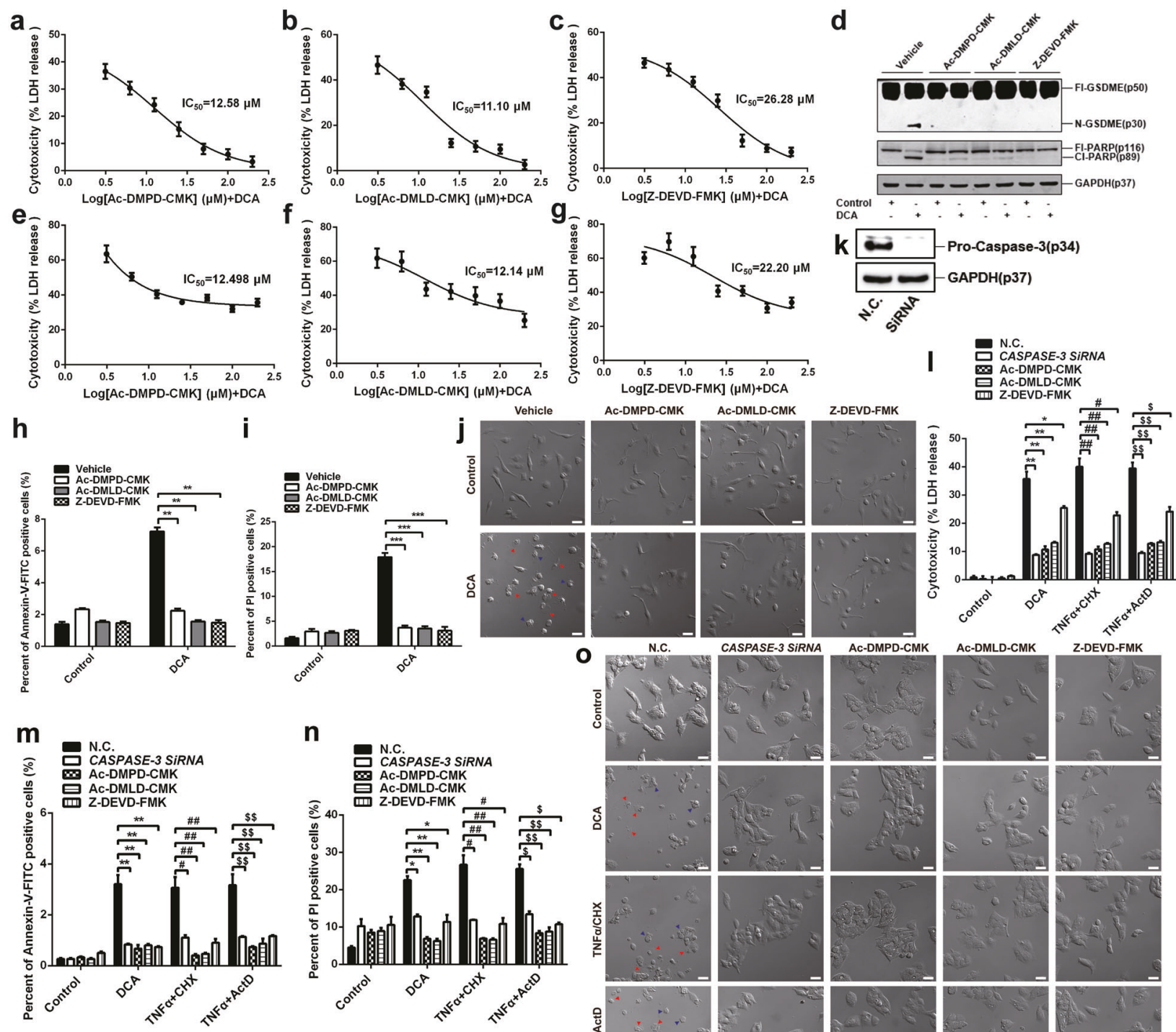

Ac-DMPD-CMK
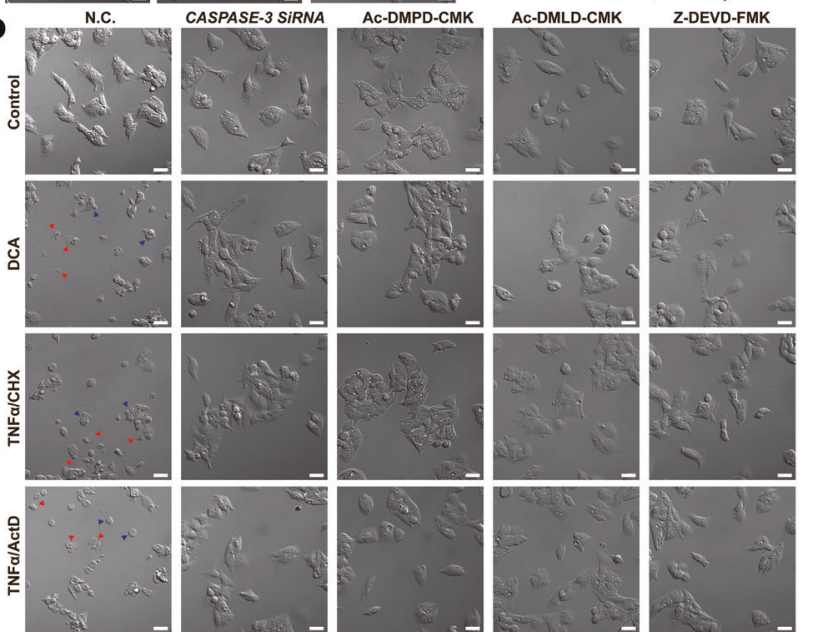

Fig. 6 Ac-DMPD/DMLD-CMK inhibited HepG2/BMDM cell death induced by bile acid. a-c LDH release assays for the cytotoxicity of HepG2 cells. $\mathbf{d}$ Representative immunoblots of GSDME and PARP in HepG2 cells. $\mathbf{e}-\mathbf{g}$ LDH release assays for the cytotoxicity of mouse BMDMs. $\mathbf{h}$ and i Flow cytometry analysis of Annexin V-FITC- or PI-positive BMDMs. $\mathbf{j}$ Changes in BMDM cell morphology were observed with a microscope (scale bar, $20 \mu \mathrm{m}$, magnification of the microphotograph, $\times 200$ ). k Silencing efficiency of CASPASE-3 in HepG2 cells. I LDH release assays for the cytotoxicity of HepG2 cells treated with inhibitors or CASPASE-3 siRNA. $\mathbf{m}$ and $\mathbf{n}$ Flow cytometry analysis of Annexin V-FITC- or PI-positive HepG2 cells treated as indicated. o Changes in HepG2 cell morphology treated as indicated were observed with a microscope (scale bar, $20 \mu \mathrm{m}$, magnification of the microphotograph, $\times 200)$. Data are expressed as the mean $\pm \mathrm{SEM}(n=3)$. ${ }^{*} P<0.05,{ }^{* *} P<0.01,{ }^{* * *} P<0.001$ vs. the vehicle group of DCA; ${ }^{\#} P<0.05,{ }^{\# \#} P<0.01$ vs. the vehicle group of TNF $\alpha / C H X ;{ }^{\$} P<0.05,{ }^{\$} P<0.01$ vs. the vehicle group of TNF $\alpha / A c t D$.

\section{ADDITIONAL INFORMATION}

The online version of this article (https://doi.org/10.1038/s41401-020-0434-2) contains supplementary material, which is available to authorized users.

Competing interests: The authors declare no competing interests.

\section{REFERENCES}

1. Balvers K, Wirtz MR, Van DS, Goslings JC, Juffermans NP. Risk factors for traumainduced coagulopathy- and transfusion-associated multiple organ failure in severely injured trauma patients. Front Med. 2015;2:24-34.

2. Hutchings $L$, Watkinson $P$, Young JD, Willett K. Defining multiple organ failure after major trauma: A comparison of the Denver, Sequential Organ Failure
Assessment, and Marshall scoring systems. J Trauma Acute Care Surg. 2017;82: 534-41.

3. Yamada N, Sanada Y, Okada N, Wakiya T, Ihara Y, Urahashi T, et al. Successful rescue of disseminated varicella infection with multiple organ failure in a pediatric living donor liver transplant recipient: a case report and literature review. Virol J. 2015;12:91-6.

4. Stryjewski ME, LiPuma JJ, Messier RH Jr., Reller LB, Alexander BD. Sepsis, multiple organ failure, and death due to Pandoraea pnomenusa infection after lung transplantation. J Clin Microbiol. 2003;41:2255-7.

5. Wang H, Lu SC, He L, Dong JH. A study on risk factors and diagnostic efficiency of posthepatectomy liver failure in the nonobstructive jaundice. Medicine. 2018;97: e9963-9.

6. Pares A, Deulofeu R, Cisneros L, Escorsell A, Salmeron JM, Caballeria J, et al Albumin dialysis improves hepatic encephalopathy and decreases circulating 
phenolic aromatic amino acids in patients with alcoholic hepatitis and severe liver failure. Crit Care. 2009;13:R8-15.

7. Valerio C, Theocharidou E, Davenport A, Agarwal B. Human albumin solution for patients with cirrhosis and acute on chronic liver failure: beyond simple volume expansion. World J Hepatol. 2016;8:345-54.

8. Li H, Zhao XK, Cheng YJ, Zhang Q, Wu J, Lu S, et al. Gasdermin D-mediated hepatocyte pyroptosis expands inflammatory responses that aggravate acute liver failure by upregulating monocyte chemotactic protein 1/CC chemokine receptor-2 to recruit macrophages. World J Gastroenterol. 2019;25:6527-40.

9. Dostert C, Petrilli V, Van BR, Steele C, Mossman BT, Tschopp J. Innate immune activation through Nalp3 inflammasome sensing of asbestos and silica. Science. 2008;320:674-7.

10. Shi J, Zhao Y, Wang Y, Gao W, Ding J, Li P, et al. Inflammatory caspases are innate immune receptors for intracellular LPS. Nature. 2014;514:187-92.

11. Lamkanfi M. Emerging inflammasome effector mechanisms. Nat Rev Immunol. 2011;11:213-20.

12. Fink SL, Cookson BT. Caspase-1-dependent pore formation during pyroptosis leads to osmotic lysis of infected host macrophages. Cell Microbiol. 2006;8: 1812-25.

13. Strasser A, Cory S, Adams JM. Deciphering the rules of programmed cell death to improve therapy of cancer and other diseases. EMBO J. 2011;30:3667-83.

14. Wang $Y$, Gao W, Shi X, Ding J, Liu W, He H, et al. Chemotherapy drugs induce pyroptosis through caspase-3 cleavage of a gasdermin. Nature. 2017;547:99-103.

15. Rogers C, Fernandes-Alnemri T, Mayes L, Alnemri D, Cingolani G, Alnemri ES. Cleavage of DFNA5 by caspase-3 during apoptosis mediates progression to secondary necrotic/pyroptotic cell death. Nat Commun. 2017:8:14128-41.

16. Yang J, Liu ZH, Wang CP, Yang R, Rathkey JK, Pinkard OW, et al. Mechanism of gasdermin $\mathrm{D}$ recognition by inflammatory caspases and their inhibition by a gasdermin D-derived peptide inhibitor. Proc Natl Acad Sci USA. 2018;115: 6792-7.

17. Lossi L, Castagna C, Merighi A. Caspase-3 mediated cell death in the normal development of the mammalian cerebellum. Int J Mol Sci. 2018;19:3999-4021.

18. Rogers C, Erkes DA, Nardone A, Aplin AE, Fernandes AT, Alnemri ES. Gasdermin pores permeabilize mitochondria to augment caspase-3 activation during apoptosis and inflammasome activation. Nat Commun. 2019;10:1689-705.

19. Shi S, Verstegen MA, Mezzanotte L, Jonge J, Lowik C, Laan LJW. Necroptotic cell death in liver transplantation and underlying diseases: mechanisms and clinical perspective. Liver Transpl. 2019;25:1091-104.

20. Hotchkiss RS, Chang KC, Swanson PE, Tinsley KW, Hui JJ, Klender P, et al. Caspase inhibitors improve survival in sepsis: a critical role of the lymphocyte. Nat Immunol. 2000;1:496-501.

21. Cheng Y, Deshmukh M, D'Costa A, Demaro JA, Gidday JM, Shah A, et al. Caspase inhibitor affords neuroprotection with delayed administration in a rat model of neonatal hypoxic-ischemic brain injury. J Clin Invest. 1998;101:1992-9.

22. Slee EA, Zhu H, Chow SC, MacFarlane M, Nicholson DW, Cohen GM. Benzyloxycarbonyl-Val-Ala-Asp (OMe) fluoromethylketone (Z-VAD.FMK) inhibits apoptosis by blocking the processing of CPP32. Biochem J. 1996;315(Pt 1):21-4.

23. Garcia CM, Peterson EP, Leiting B, Ruel R, Nicholson DW, Thornberry NA. Inhibition of human caspases by peptide-based and macromolecular inhibitors. J Biol Chem. 1998;273:32608-13.

24. Nicholson DW, Ali A, Thornberry NA, Vaillancourt JP, Ding CK, Gallant M, et al. Identification and inhibition of the ICE/CED-3 protease necessary for mammalian apoptosis. Nature. 1995:376:37-43.

25. Meguro T, Chen B, Parent AD, Zhang JH. Caspase inhibitors attenuate oxyhemoglobin-induced apoptosis in endothelial cells. Stroke. 2001;32:561-6.

26. Zhang X, Goncalves R, Mosser DM. The isolation and characterization of murine macrophages. Curr Protoc Immunol. 2008;Chapter 14:Unit 14 1-18.

27. Hao H, Cao L, Jiang C, Che Y, Zhang S, Takahashi S, et al. Farnesoid X receptor regulation of the NLRP3 inflammasome underlies cholestasis-associated sepsis. Cell Metab. 2017;25:856-867 e5.

28. Feeney B, Pop C, Swartz P, Mattos C, Clark ACJB. Role of loop bundle hydrogen bonds in the maturation and activity of (Pro) caspase-3. Biochemistry. 2006; 45:13249-63.
29. Morris GM, Huey R, Lindstrom W, Sanner MF, Belew RK, Goodsell DS, et al. AutoDock4 and AutoDockTools4: automated docking with selective receptor flexibility. J Comput Chem. 2009;30:2785-91.

30. Morris GM, Goodsell DS, Halliday RS, Huey R, Hart WE, Belew RK, et al. Automated docking using a Lamarckian genetic algorithm and an empirical binding free energy function. J Comput Chem. 1998;19:1639-62.

31. Sun HY, Ji FQ, Fu LY, Wang ZY, Zhang HY. Structural and energetic analyses of SNPs in drug targets and implications for drug therapy. J Chem Inf Model. 2013;53:3343-51.

32. Sun HY, Hou TJ, Zhang HY. Finding chemical drugs for genetic diseases. Drug Discov Today 2014;19:1836-40.

33. Hou X, Du J, Zhang J, Du L, Fang H, Li M. How to improve docking accuracy of AutoDock4.2: A case study using different electrostatic potentials. J Chem Inf Model. 2013;53:188-200.

34. Gasteiger J, Marsili M. Iterative partial equalization of orbital electronegativity-a rapid access to atomic charges. Tetrahedron. 1980;36:3219-28.

35. Ekert PG, Silke J, Vaux DL. Caspase inhibitors. Cell Death Differ. 1999;6:1081-6.

36. Thomsen ND, Koerber JT, Wells JA. Structural snapshots reveal distinct mechanisms of procaspase-3 and-7 activation. Proc Natl Acad Sci USA. 2013;110: 8477-82.

37. Yang J, Liu ZH, Wang CP, Yang R, Rathkey JK, Shi WX, et al. Mechanism of gasdermin $D$ recognition by inflammatory caspases and their inhibition by a gasdermin D-derived peptide inhibitor. FASEB J. 2019;115:33-9.

38. Giroux A. Discovery of novel aspartyl ketone dipeptides as potent and selective caspase-3 inhibitors. Abstr Pap Am Chem S. 2005;230:U3315-U3315.

39. Wang Y, Liu X, Zhang D, Chen J, Liu S, Berk M. The effects of apoptosis vulnerability markers on the myocardium in depression after myocardial infarction. BMC Med. 2013;11:32-40.

40. Heymann F, Tacke F. Immunology in the liver-from homeostasis to disease. Nat Rev Gastroenterol. Hepatol. 2016;13:88-110.

41. Anastasiou O, Sydor S, Sowa JP, Manka P, Katsounas A, Syn WK, et al. Higher thyroid-stimulating hormone, triiodothyronine and thyroxine values are associated with better outcome in acute liver failure. PLoS One 2015;10: e0132189-201.

42. Masubuchi N, Sugihara M, Sugita T, Amano K, Nakano M, Matsuura T. Oxidative stress markers, secondary bile acids and sulfated bile acids classify the clinical liver injury type: Promising diagnostic biomarkers for cholestasis. Chem-Biol Interact. 2016;255:83-91.

43. Zhang L, Cheng Y, Du X, Chen S, Feng X, Gao Y, et al. Swertianlarin, an herbal agent derived from swertia mussotii franch, attenuates liver injury, inflammation, and cholestasis in common bile duct-ligated rats. Evid Based Complement Altern Med. 2015;2015:1-10.

44. Sousa T, Castro RE, Pinto SN, Coutinho A, Lucas SD, Moreira R, et al. Deoxycholic acid modulates cell death signaling through changes in mitochondrial membrane properties. J Lipid Res. 2015;56:2158-71.

45. Kabbani AR, Tergast TL, Manns MP, Maasoumy B. Treatment strategies for acuteon-chronic liver failure. Med Klin Intensivmed Notfmed. 2019;28:1-13.

46. Kristiansen RG. Current state of knowledge of hepatic encephalopathy (part I): newer treatment strategies for hyperammonemia in liver failure. Metab Brain Dis. 2016;31:1357-8

47. Volarevic V, Nurkovic J, Arsenijevic N, Stojkovic M. Concise review: therapeutic potential of mesenchymal stem cells for the treatment of acute liver failure and cirrhosis. Stem Cells. 2014;32:2818-23.

48. Stockmann HB, Jn IJ. Prospects for the temporary treatment of acute liver failure. Eur J Gastroenterol Hepatol. 2002;14:195-203.

49. Luedde T, Kaplowitz N, Schwabe RF. Cell death and cell death responses in liver disease: mechanisms and clinical relevance. Gastroenterology. 2014;147: 765-U110.

50. Tsuchida T, Friedman SL. Mechanisms of hepatic stellate cell activation. Nat Rev Gastro Hepat. 2017;14:397-411.

51. Saporito MS, Hudkins RL, Maroney AC. Discovery of CEP-1347/KT-7515, an inhibitor of the JNK/SAPK pathway for the treatment of neurodegenerative diseases. Prog Med Chem. 2002;40:23-62. 